\title{
Estudio de adsorción, retención y difusión de pesticidas piretroides como la permetrina en suelo agrícola y su capacidad para actuar como filtro
}

\section{Study of adsorption, retention and diffusion of pyrethroid pesticides such as permethrin in agricultural land and its ability to act as a filter}

\author{
GODINEZ-GARCÍA, Andrés† $\dagger$, DÍAZ-TECANHUEY, Pedro Jesús, GUIJOSA-GUADARRAMA, \\ Santiago y HERNÁNDEZ-MORALES, María Guadalupe
}

Universidad Autónoma Metropolitana- Azcapotzalco. Departamento de Ciencias Básicas

ID $1^{\text {er }}$ Autor: Andres, Godinez-García / ORC ID: 0000-0002-0732-5591, CVU CONACYT ID: 168578

ID $1^{\text {er }}$ Coautor: Pedro Jesús, Díaz-Tecanhuey / ORC ID: 0000-0002-8064-3684

ID $2^{\text {do }}$ Coautor: Santiago, Guijosa-Guadarrama / ORC ID: 0000-0003-0391-6332

ID $3^{\text {er }}$ Coautor: María Guadalupe, Hernández-Morales / ORC ID: 0000-0002-6793-273X, CVU CONACYT ID: 74002

DOI: $10.35429 / J U S D .2019 .17 .5 .20 .26$

Recibido: 30 de Agosto, 2019; Aceptado 30 de Diciembre, 2019

\begin{abstract}
Resumen
El presente trabajo se inscribe dentro de una de las prioridades del desarrollo sostenible que es la conservación del suelo y el cuidado de los mantos acuíferos. Es conocido que el suelo actúa como filtro natural de contaminantes del agua subterránea, por ello uno de los objetivos de mayor relevancia es entender la interacción que existe entre el suelo y los pesticidas, en especial los piretroides que son unos de los más utilizados actualmente, así como el proceso de retención y difusión de estas sustancias a través del suelo. Se realizó un estudio sistemático para determinar la transferencia, difusión y retención de pesticidas piretroides a través de muestras de suelo agrícola. Se identificaron los tipos de suelo a utilizar en el estudio, se determinó de forma cualitativa la cantidad de grupos funcionales utilizando Espectroscopía Infrarroja por la Transformada de Fourier (FTIR) con Reflexión Total Atenuada (ATR). Se tomó como molécula modelo de piretroide la permetrina, la concentración cualitativa de permetrina absorbida en un lecho de suelo se realizó monitoriando la absorbancia de una suspensión de permetrina en agua. Se muestra la capacidad de retención de pesticida de dos tipos de suelos húmedos y se determina el flujo másico de suspensión dentro del lecho de suelo. La magnitud del flujo másico se relacionó inversamente a la cantidad de grupos funcionales del suelo.
\end{abstract}

Difusión, Flux, Pesticidas, Piretroide, Suelo

\begin{abstract}
This work is part of one of the priorities of sustainable development that is the conservation of soil and the care of aquifers. It is known that soil acts as a natural filter for contaminants in groundwater, so one of the most important objectives is to understand the interaction between soil and pesticides, especially pyrethroids, which are some of the most widely used today. as well as the process of retention and diffusion of these substances through the soil. A systematic study was carried out to determine the transfer, diffusion and retention of pyrethroid pesticides through agricultural soil samples. The kind of soils to be used in this study were identified, the quantity of functional groups was determined qualitatively using Infrared Spectroscopy by the Fourier Transform (FTIR) with Attenuated Total Reflection (ATR). Permethrin was taken as the pyrethroid model molecule, the qualitative concentration of permethrin absorbed in a soil bed was monitored using the absorbance of a suspension of permethrin in water. The pesticide retention capacity of two types of wet soil is shown and the mass flow through the soil bed was determined too. The magnitude of the mass flow was inversely related to the amount of functional groups in the soil.
\end{abstract}

Diffusion, Flux, Pesticides, Pyrethroid, Soil

Citación: GODINEZ-GARCÍA, Andrés, DÍAZ-TECANHUEY, Pedro Jesús, GUIJOSA-GUADARRAMA, Santiago y HERNÁNDEZ-MORALES, María Guadalupe. Estudio de adsorción, retención y difusión de pesticidas piretroides como la permetrina en suelo agrícola y su capacidad para actuar como filtro. Revista del Desarrollo Urbano y Sustentable. 2019. 517: $20-26$

$\dagger$ Investigador contribuyendo como primer autor 


\section{Introducción}

Una de las prioridades del desarrollo sostenible es la conservación del suelo y el cuidado de los mantos acuíferos. Es bien conocido que el suelo actúa como filtro natural de contaminantes del agua subterránea. Sin embargo, el uso cada vez mayor de sustancias químicas como los pesticidas, en el caso de la agricultura, llega a contaminar grandes cantidades de agua (Daga et al. 2019, Fonseca et al. 2019, Olave 2019, Ortiz 2019, Moore et. al. 2018). Es por ello que uno de los objetivos de mayor relevancia busca entender la interacción que existe entre el suelo y los pesticidas, en especial los peritroides que son los que más se utilizan actualmente (Xiao et al. 2018), así como el proceso de retención y difusión de estas sustancias a través del suelo (Amaroli et. al. 2018, Bai et. al. 2007, Bronshtein et. al. 2012, Libardi et. al. 1982).

El entendimiento profundo de estos procesos permite la prevención oportuna de la contaminación, y no se limita a ello sino que también abre una ventana para el diseño de filtros a basados en el suelo para eliminar estas sustancias químicas una vez que ya se encuentran en el agua y evitar que lleguen al consumo humano. En especial, los pesticidas piretroides son pesticidas artificiales desarrollados para controlar las plagas de insectos. Son una de las principales armas elegidas por los productores agropecuarios. $\mathrm{Su}$ acción, como el de los demás insecticidas, es a nivel sistema nervioso, generando una alteración de la transmisión del impulso nervioso. Su efecto fundamental se debe a una modificación en el canal del sodio de la membrana nerviosa. Los piretroides entran al ambiente principalmente debido a su uso como insecticidas (Yang et al. 2004). En el aire son degradados en unos días por la luz o por otros compuestos que se encuentran en la atmósfera. Son muy poco solubles en agua y dependiendo de las características superficiales del suelo pueden quedar retenidos en las capas superficiales.

Dependiendo del tipo de suelo se tienen diferentes grupos funcionales en la superficie lo que le dan al suelo propiedades hidrófilas (Woche et al. 2017). En México los tipos de suelo más comunes son: regosol, litosol, xerosol, yermosol, cambisol, rendzina, vertisol y feozem (INEGI 2019).
Los cuales poseen una composición diferente tanto estructural como superficialmente. En este caso se estudian las propiedades físicas y químicas del suelo tipo vertisol y feozem, que se encuentran en la región del Bajío Guanajuato y Estado de México, respectivamente y su relación con la retención o difusión de pesticidas piretroides como la permetrina. Estos suelos poseen un valor muy alto para la industria agrícola del país ya que se encuentra en unas de las regiones agrícolas más importantes de México.

El estudio de las propiedades fisicoquímicas de los suelos son de mucho interés porque con ello se puede predecir su capacidad para retener sustancias en la superficie o para dejarlas pasar hasta llegar a los mantos acuíferos por lo que se pueden hacer predicciones sobre los pesticidas que se utilizan en los cultivos del suelo. Se realiza un estudio sistemático donde se desarrolla una metodología que involucra cuestiones medulares relacionadas con: Retención, transferencia y difusión a través de suelo agrícola de la zona de Toluca Estado de México y del Bajío Guanajuato para establecer los factores que rigen la relación fundamental entre las características del suelo y el transporte de estas sustancias químicas a través de él. Estos resultados dan indicio para diseñar filtros naturales similar a como el suelo actúa, para el tratamiento de grandes cantidades de agua con menor costo respecto a métodos convencionales, lo cual es un pequeño paso para entender como conservar las reservas de agua nacionales sin contaminar.

\section{Metodología a desarrollar}

Las muestras de suelo que se utilizaron fueron tomadas de dos de las regiones agrícolas más importantes en México, la cuáles son el Bajío Guanajuato y cerca de Toluca dentro del Estado de México. Los suelos se caracterización utilizando Espectroscopía Infrarroja por la Transformada de Fourier (FTIR) con Reflexión Total Atenuada (ATR) para ello se usó un instrumento Perkin Elmer Spectrum Gx FT-IR instrument en el intervalo de 600 to $4000 \mathrm{~cm}^{-1}$.

Mientras que para hacer el análisis de la absorción de permetrina en el suelo, se preparó una solución de $250 \mathrm{mg}$ permetrina $/ 5 \mathrm{ml}$ hexano, y de esta se tomó $0.5 \mathrm{ml}$ con la que se formó una emulsión dispersándolo en $200 \mathrm{ml}$ de $\mathrm{H}_{2} \mathrm{O}$, esta se nombró solución madre.

GODINEZ-GARCÍA, Andrés, DÍAZ-TECANHUEY, Pedro Jesús, GUIJOSA GUADARRAMA, Santiago y HERNÁNDEZ-MORALES, María Guadalupe. Estudio de adsorción, retención y difusión de pesticidas piretroides como la permetrina en suelo agrícola y su capacidad para actuar como filtro. Revista del Desarrollo Urbano y Sustentable. 2019 
Posteriormente en dos buretas se colocaron las muestras de suelo del bajío y del estado de México en cada una. El suelo en polvo fino, se tamizó con una malla de 0.3 x 0.3 mm, se agregó lentamente hasta ocupar $10 \mathrm{ml}$ de la bureta, en la parte superior se añadió $7 \mathrm{ml}$ de la solución madre de permetrina y se comenzó a contar el tiempo y a medir el volumen que se iba absorbiendo en cada uno de los suelos. Esta primera parte del experimento (región A) se llevó a cabo con la bureta cerrada, una vez que todo el suelo se humedeció ocupando los intersticios de las partículas de suelo se abrió la válvula de la bureta y se continuó midiendo el flujo volumétrico (región B). Para determinar de forma cualitativa la cantidad de permetrina que se absorbe en el suelo se mide la absorbancia de la solución madre remanente que quedó sobre la muestra de suelo, una vez que se humedeció completamente el suelo, durante la primera parte del experimento. Esta prueba se realizó con un espectrofotómetro Shimadzu UV-160 A.

\section{Resultados}

En México se poseen varios tipos de suelo entre los que se encuentran: el regosol, litosol, xerosol, yermosol, cambisol, rendzina, vertisol, feozem. (INEGI 2019) De las coordenadas donde se tomaron las muestras (figura 1) y por datos de la INEGI, se encuentra que el suelo del Estado de México es del tipo feozem, mientras que el tomado del Bajío es una mezcla de feozem y vertisol.

El suelo tipo vertisol tiene un alto contenido de arcilla (> 30\%), la cual se expande $\mathrm{y}$ se vuelve pegajoso cuando se encuentra en humedad $\mathrm{y}$, en seco forma grietas anchas $\mathrm{y}$ profundas. Mientras que el suelo tipo feozem, es un suelo que posee una capa superficial oscura, suave, rica en materia orgánica y en nutrientes. Estos dos tipos de suelos al poseer propiedades diferentes, también es de esperarse que se observen características diferentes en cuanto al transporte de la solución madre a través de él. En los experimentos se procuró tener un tamaño de partícula homogéneo y similar entre ambos suelos para que la porosidad sea también similar, ya que esta tiene un efecto considerable en las propiedades de transporte del fluido a través del lecho de suelo. De esta forma se aseguró que el flujo másico de la solución madre se debiera solo a las propiedades superficiales de ambos suelos.
Es conocido que las propiedades superficiales de un material, sobre todo su capacidad hidrófila e hidrófoba depende del tipo de grupos funcionales que poseen ambos suelos (Woche 2017). En la figura 2, se muestran los colores característicos de las muestras de suelo tomadas del Bajío en las coordenadas $20^{\circ} 42^{\prime} 57.58^{\prime}$ ' N, $100^{\circ} 46^{\prime} 38.50^{\prime}{ }^{\prime} \mathrm{W}$ $\mathrm{y}$ en el Edo. Mex. 19'18'38.2' 'N, 99²7'46.3''W. También, se clasifican de acuerdo al color usando el sistema Munsell. El suelo seco del Edo. Mex. Tiene una clasificación 10 YR 7/6 y húmedo 7.5 R 4/1. Mientras que el suelo del Bajío seco una clasificación 7.5 R 7/1 y el húmedo 7.5 R 4/1.
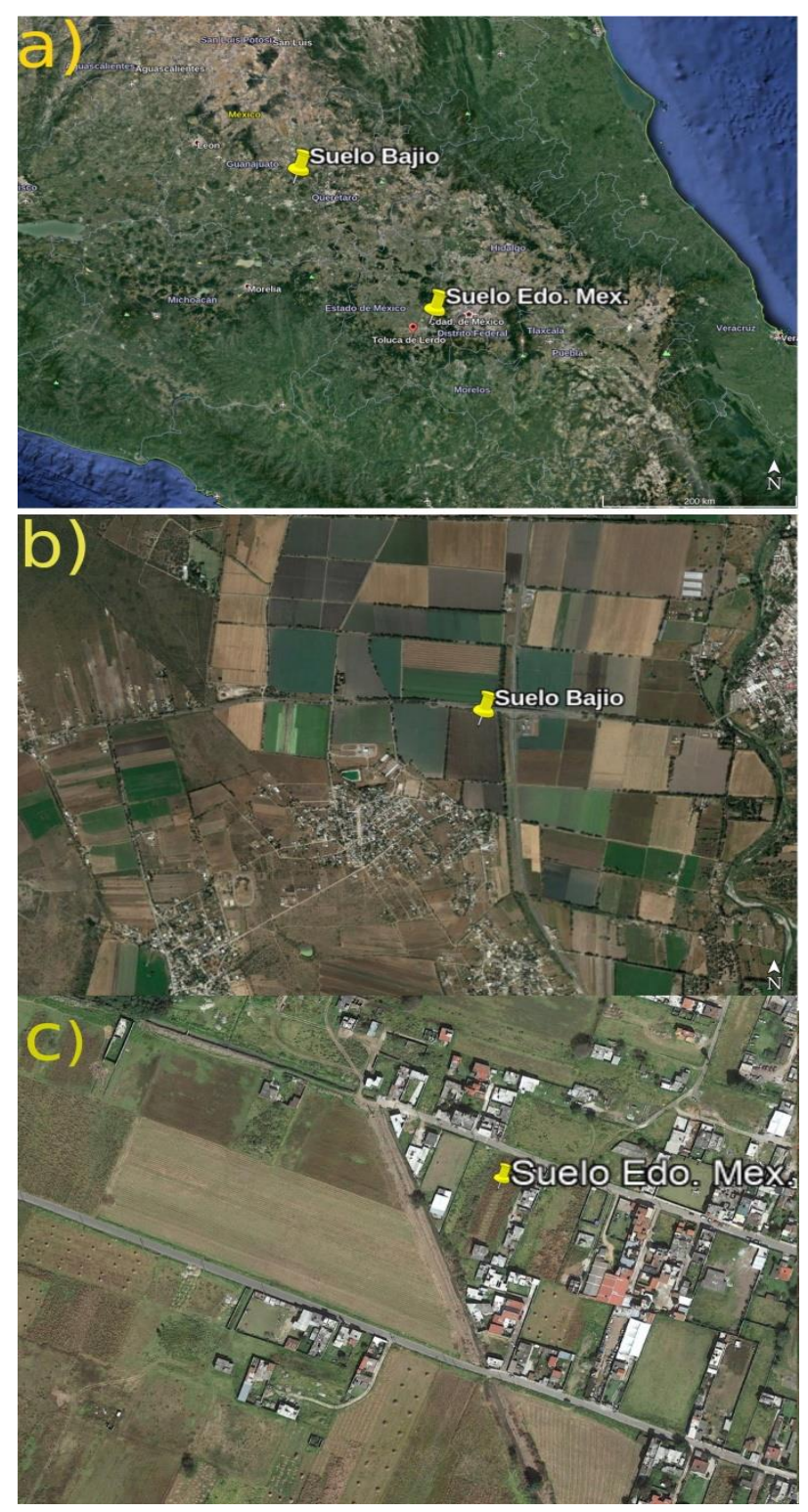

Figura 1 a) Ubicación de suelos muestreados b) Suelo Bajío $\left(20^{\circ} 42^{\prime} 57.58^{\prime \prime} \mathrm{N}\right.$ y $\left.100^{\circ} 46^{\prime} 38.50^{\prime \prime} \mathrm{W}\right)$, c) Suelo

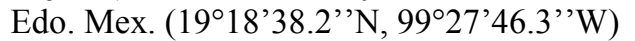

Fuente: Imágenes obtenidas de google earth. 


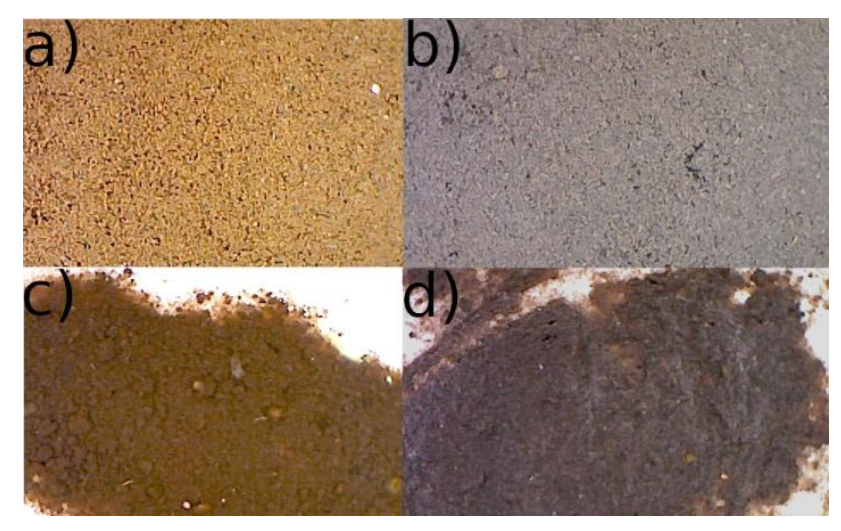

Figura 2 Tipos de suelos analizados y clasificación del color de acuerdo al sistema Munsell, a) Suelo Edo. Mex. seco color: 10 YR 7/6, b) Suelo Bajío seco color: $7.5 \mathrm{R}$ 7/1, c) Suelo Edo. Mex. húmedo color: 10 YR 4/6, d) Suelo Bajío húmedo color: 7.5 R 4/1

En el figura 3 se muestran los espectros en el infrarrojo para las muestras de suelo del Bajío y del Estado de México. Ambas muestras presentan espectros muy similares que son caracteristicos de suelo con humus (Davis \& Mauer 2010, Deng 2019), los picos en 3392 (vibraciones de estiramiento H-O), 2948 y $2873 \mathrm{~cm}^{-1}$ (vibraciones asimétricas y simétricas de grupos alifáticos, $\mathrm{CH}_{3}$ y $\left.\mathrm{CH}_{2}\right)$ (Cox et al. 2010, Parikh et al. 2014 and Six et al. 2002). El pico en $1036 \mathrm{~cm}^{-1}$ se debe a las vibraciones de estiramiento $\mathrm{Si}-\mathrm{O}$ y también otros autores han probado que se debe a la presencia de vibraciones de estiramiento $\mathrm{C}-\mathrm{O}$ de polisacáridos ambos grupos son altamente hidrófilos (Solomon 2005) y de forma cualitativa que este pico es más intenso en el suelo del bajío.

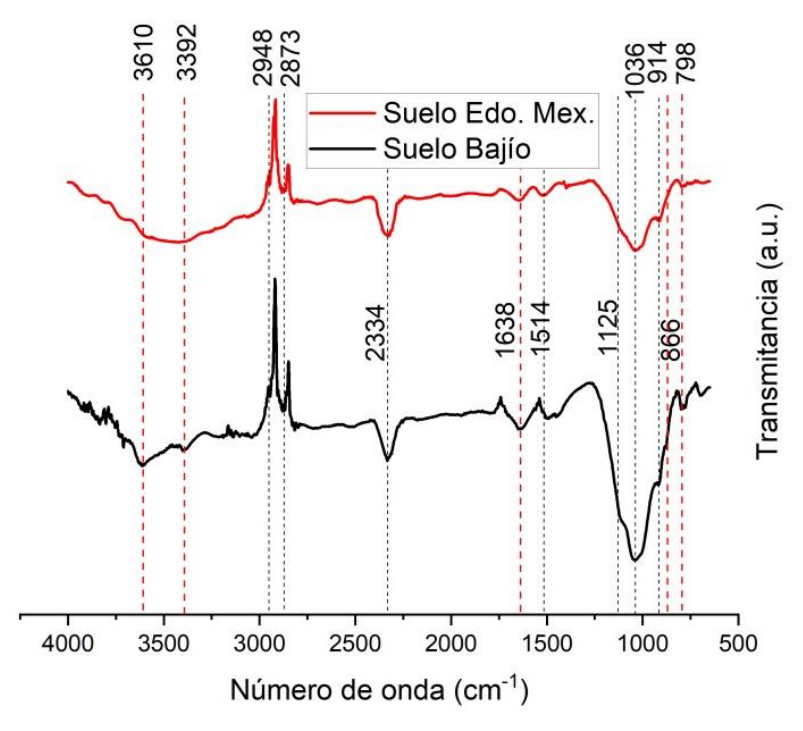

Figura 3 Spectro infrarrojo para los suelos del bajío y del Edo. del Mex

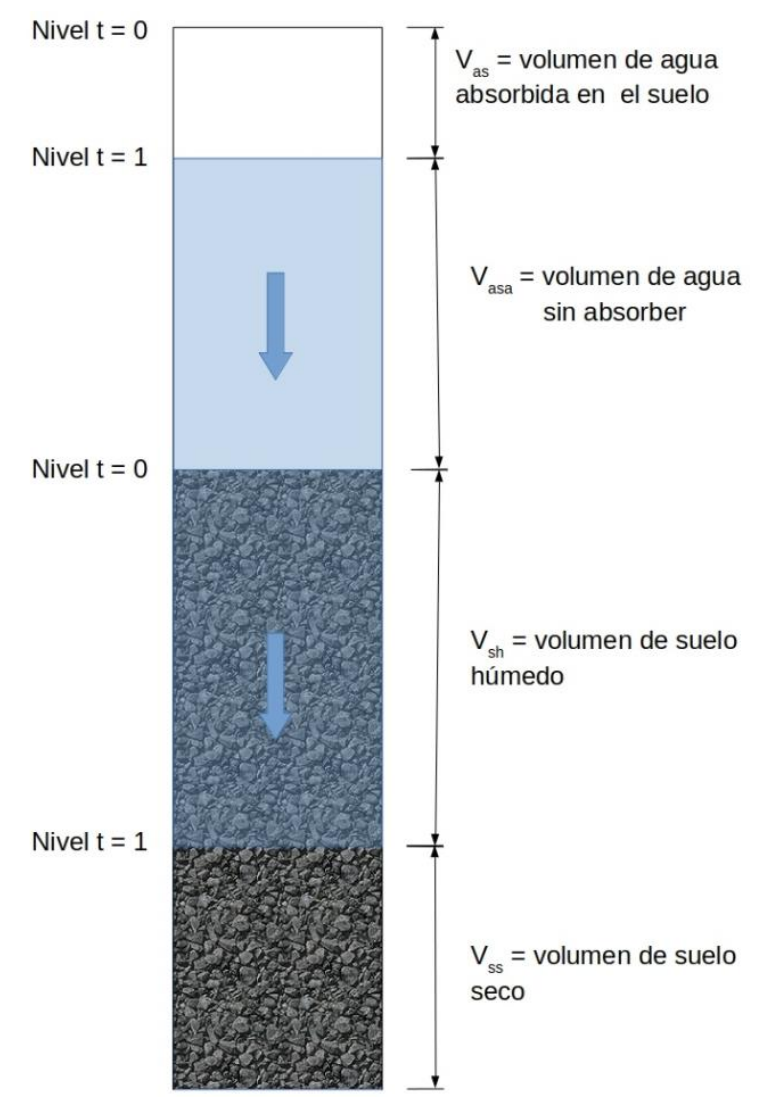

Figura 4 Fenómenos de transporte de una suspensión de pesticida en agua a través de suelo agrícola

La figura 4, representa el proceso de transferencia de la solución madre en las muestras de suelo. La solución se agrega en la parte superior del suelo. Y lentamente comienza a ser absorbida por el suelo, se monitorea el nivel de la solución remanente a medida que transcurre el tiempo, para saber la velocidad con la cuál es absorbida por el suelo. Es interesante hacer notar que en un inicio los poros de la tierra contenían aire, pero posteriormente debido a la atracción de los grupos hidrófilos el agua es atraída hacia el suelo con una intensidad tal que desplaza el aire que inicialmente se encontraba ocupando los poros. El desplazamiento del aire por la solución es muy importante porque se puede determinar de una forma simple la porosidad, dividiendo el volumen de agua absorbida entre el volumen que ocupa el suelo húmedo.

$\%$ Porosidad $=\frac{V_{a s}}{V_{s h}} * 100$

La nomenclatura de la ecuación 1 está definida en la figura 4. En la tabla 1 se presentan los valores del porcentaje de porosidad obtenida para ambos suelos estudiados. Se puede observar que los valores son muy similares como era algo esperado ya que ambos se tamizaron con una malla del mismo tamaño.

GODINEZ-GARCÍA, Andrés, DÍAZ-TECANHUEY, Pedro Jesús, GUIJOSAGUADARRAMA, Santiago y HERNÁNDEZ-MORALES, María Guadalupe. Estudio de adsorción, retención y difusión de pesticidas piretroides como la permetrina en suelo agrícola y su capacidad para actuar como filtro. Revista del permetrina en suelo agrícola y su capacidad para actuar como filtro. Revista del
Desarrollo Urbano y Sustentable. 2019 


\begin{tabular}{|l|r|r|r|}
\hline Suelo & $\begin{array}{c}\text { Porosidad } \\
\text { [\%] }\end{array}$ & \multicolumn{1}{c|}{$\begin{array}{c}\text { Flujo } \\
\text { másico } \\
\text { región (A) } \\
{\left[\mathrm{Kg} / \mathrm{m}^{2} \mathrm{~h}\right]}\end{array}$} & $\begin{array}{c}\text { Flujo } \\
\text { másico } \\
\text { Región (B) }\end{array}$ \\
\hline $\begin{array}{l}\text { Edo. } \\
\text { Mex. }\end{array}$
\end{tabular}

Tabla 1 Porosidad de los suelos y flujo másico promedio obtenido de la figura 5

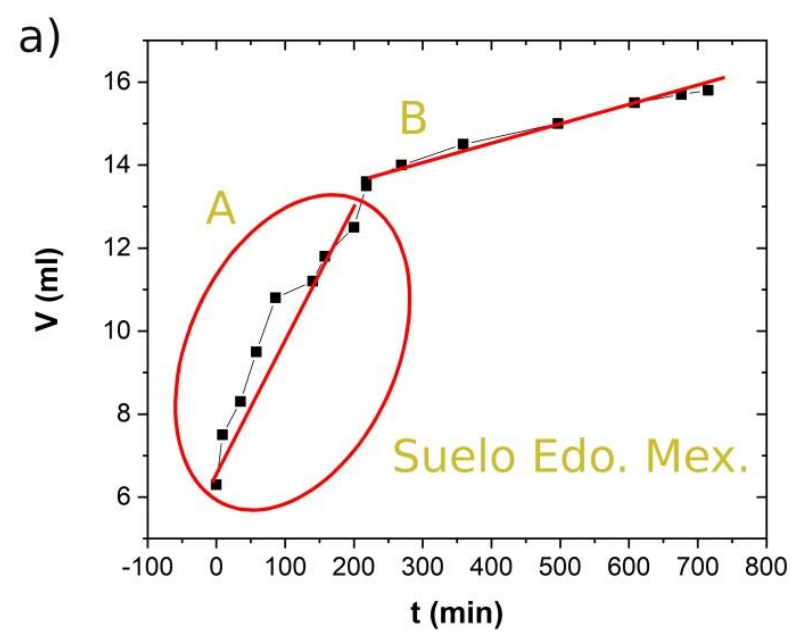

b)

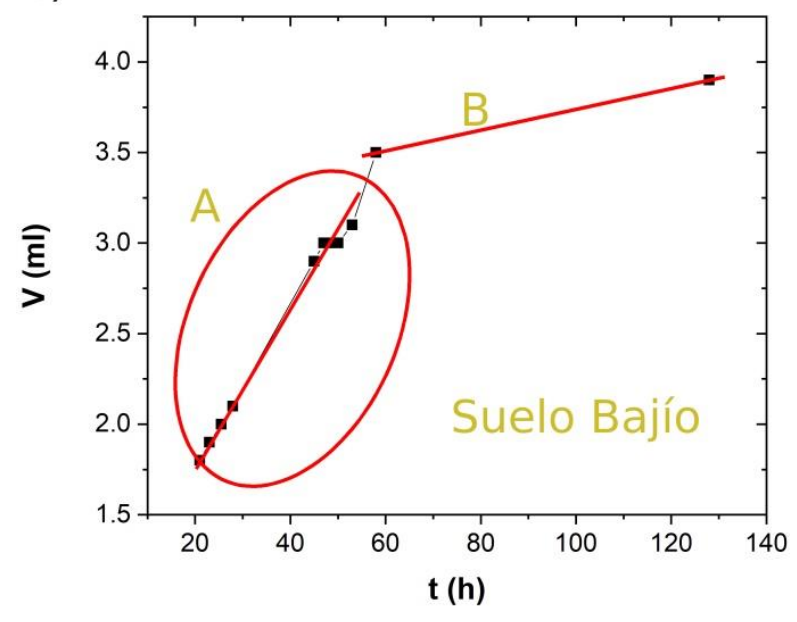

Grafico 1 Volumen de la solución madre permeada en los suelos a través del tiempo. a) Suelo del Edo. Mex. y b) Suelo del Bajío

En el Grafico 1, se muestran la variación del volumen del agua absorbida a través del tiempo. La pendiente de la región A se debe al flujo másico que se va absorbiendo en la tierra a medida que se va humedeciendo. Una vez humedecida se puede observar la región $\mathrm{B}$, donde se observa que el flujo másico disminuye considerablemente (Tabla 1). Esto último se debe a que el suelo posee grupos hidrófilos que atraen el agua y una vez que se humedece, el flujo másico disminuye debido a que todos los grupos funcionales se unieron a las moléculas de agua y la fuerza de gravedad no es lo suficiente intensa como para vencer esta fuerza de atracción.
De acuerdo al espectro infrarrojo es de esperarse que el suelo del bajío atraiga con mayor fuerza al agua debido a que la señal característica de los grupos funcionales $\mathrm{Si}-\mathrm{O}$ y polisacáridos altamente hidrófilos es más intensa.

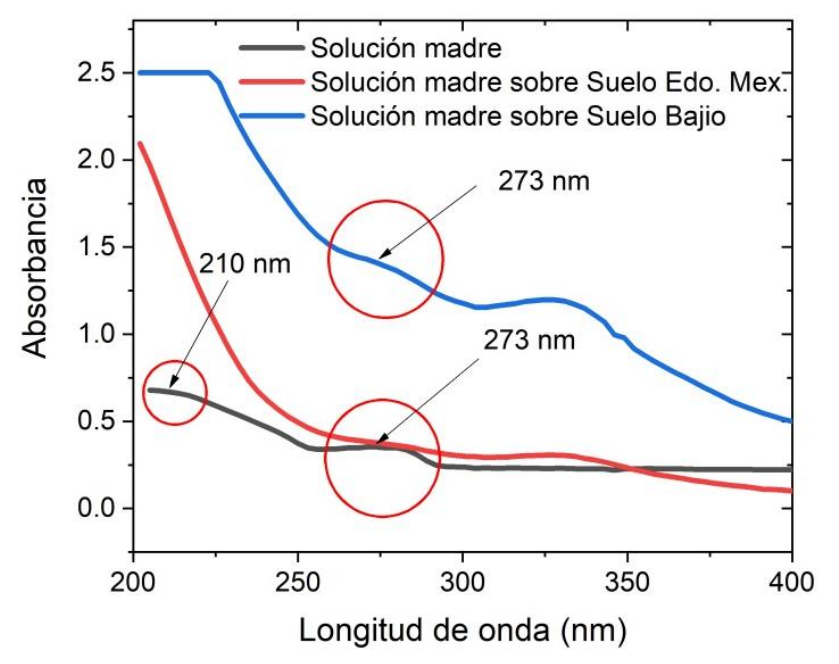

Grafico 2 Espectroscopia UV-Vis realizada sobre, la solución madre (-), remanente de la solución madre agregada sobre el suelo del Edo. Mex. (-) y remanente de la solución madre agregada sobre el suelo del Bajío (-)

En la Figura 5 se muestran los espectros UV-Vis de la solución madre remanente agregada sobre suelo del Edo. Mex y del Bajío después de que parte de esta se absorbió hasta humedecerlo completamente y llenar los intersticios entre partículas. Se puede observar en la figura que cuando se absorbe la solución madre en los suelos, el líquido remanente en la superficie de cada suelo presenta una absorción mayor que la de la solución madre, lo que significa el líquido remanente presenta una mayor concentración de permetrina.

Esto solo puede suceder si el pesticida queda sin absorber en el suelo, por lo que se concentra cuando parte del agua queda absorbida dentro del suelo. En el caso del suelo del Edo. Mex. al poseer menos grupos funcionales hidrófilos la fricción molecular del agua y permetrina con las partículas del suelo es menor, y como consecuencia se puede estas moléculas se pueden deslizar con mayor facilidad sobre estas, por lo que el flujo másico es mayor y parte de la permetrina penetra en el suelo. Mientras que en el caso del suelo del bajío, al poseer una mayor cantidad de grupos funcionales hidrófilos hace que la atracción sobre las partículas de suelo sea más intensa por lo que deslizarse sobre ellas es más difícil. Esto genera que el másico sea menor. 
Esto nos permite entender que la cantidad de grupos hidrófilos tendrá un efecto inverso sobre la velocidad con la que el agua penetra en el suelo y es de esperarse que también sobre su coeficiente de difusión, cuya determinación será incluida en un estudio posterior.

\section{Agradecimiento}

Se agradece al Profesor Humberto Vázquez Torres de la UAM Iztapalapa por la ayuda prestada en el espectro ATR-FTIR así como al y a los profesores del área Química y fisicoquímica ambiental de la UAM Azcapotzalco por poner a nuestra disposición el espectrofotómetro UV-Vis.

\section{Conclusiones}

Se presenta un estudio sobre la capacidad de los suelos para retener o absorber pesticidas. Se utilizó como muestras dos tipos de suelos, una de suelo del Bajío y otra del estado de México. Se tomaron espectros en el infrarrojo de los dos suelos, con lo que se encontró cualitativamente que el suelo del Bajío posee una cantidad mayor de grupos funcionales $\mathrm{Si}-\mathrm{O}$ y de polisacáridos altamente hidrófilos. Estos grupos tienen un efecto inverso sobre la velocidad con la que el agua penetra en el suelo. En cada suelo se observa que el pesticida al ser hidrofóbico se queda en el agua remanente de acuerdo a los espectros UV-Vis obtenidos. En el caso del suelo del Edo. de Mex. al poseer una menor cantidad de grupos hidrófilos, permite el paso de una mayor cantidad de pesticidas por lo que el espectro UV-Vis del líquido remanente que indica la presencia de permetrina es menos intenso que en el caso del bajío que retiene una mayor cantidad de pesticida. Un estudio posterior permitirá cuantificar el porcentaje de permetrina que se retiene y permea en ambos suelos.

\section{Referencias}

Amaroli, A., Gallus, L., \& Ferrando, S. (2018). Permethrin drastically affects the developmental cycle of the non-target slime mould Dictyostelium discoideum. Chemosphere, 193, 1-7.
Bai, J., Deng, W., Cui, B., \& Ouyang, H. (2007). Water diffusion coefficients of horizontal soil columns from natural salinealkaline wetlands in a semiarid area. Eurasian Soil Science, 40(6), 660-664.

Bronshtein, A., Chuang, J. C., Van Emon, J. M., \& Altstein, M. (2012). Development of a multianalyte enzyme-linked immunosorbent assay for permethrin and aroclors and its implementation for analysis of soil/sediment and house dust extracts. Journal of agricultural and food chemistry, 60(17), 4235-4242.

Cox, R. J., Peterson, H. L., Young, J., Cusik, C., \& Espinoza, E. O. (2000). The forensic analysis of soil organic by FTIR. Forensic science international, 108(2), 107-116.

Daga, D. Y., Zulaica, L., \& Vazquez, P. (2019). Plaguicidas en el Cinturón Hortícola Marplatense, Buenos Aires, Argentina: servicios ecosistémicos comprometidos y estrategias de manejo/Plaguicides in the Marplatense Horticultural Belt, Buenos Aires, Argentina: ecosystem services compromised and management strategies. Caderno de Geografia, 29(56), 98.

Davis, R., \& Mauer, L. J. (2010). Fourier transform infrared (FT-IR) spectroscopy: a rapid tool for detection and analysis of foodborne pathogenic bacteria. Current research, technology and education topics in applied microbiology and microbial biotechnology, 2, 1582-1594.

Deng, J., Zhu, W., Zhou, Y., \& Yin, Y. (2019). Soil Organic Carbon Chemical Functional Groups under Different Revegetation Types Are Coupled with Changes in the Microbial Community Composition and the Functional Genes. Forests, 10(3), 240.

Fonseca Hernández, L. R., \& Celis Forero, A. (2019). PRACTICAS DE MANEJO DE NUEVOS GENOTIPOS DE HABICHUELA (Phaseolus vulgaris L.) TIPO VOLUBLE EN LA REGIÓN DEL SUMAPAZ.

INEGI

(2019).

Edafología.

México: www.inegi.org.mx.

https://www.inegi.org.mx/temas/edafologia/. 
Libardi, P. L., Reichardt, K., Jose, C., Bazza, M., \& Nielsen, D. R. (1982). An approximate method of estimating soil water diffusivity for different soil bulk densities. Water Resources Research, 18(1), 177-181.

Moore, M. T., Locke, M. A., \& Cullum, R. F. (2018). Expanding Wetland Mitigation: Can Rice Fields Remediate Pesticides in Agricultural Runoff?. Journal of environmental quality.

Olave Seguel, C. E. (2019). Estudio de adsorción de plaguicidas en sedimentos fluviales y optimización de técnicas de recuperación.

Ortiz Huamani, H. (2019). Abonamiento orgánico y químico en el cultivo de brócoli (Brassica oleracea L.) en la comunidad campesina de Los Ángeles, HuancaramaAndahuaylas-Apurímac.

Parikh, S. J., Goyne, K. W., Margenot, A. J., Mukome, F. N., \& Calderón, F. J. (2014). Soil chemical insights provided through vibrational spectroscopy. In Advances in agronomy (Vol. 126, pp. 1-148). Academic Press.

Six, J., Conant, R. T., Paul, E. A., \& Paustian, K. (2002). Stabilization mechanisms of soil organic matter: implications for C-saturation of soils. Plant and soil, 241(2), 155-176.

Solomon, D., Lehmann, J., Kinyangi, J., Liang, B., \& Schäfer, T. (2005). Carbon K-edge NEXAFS and FTIR-ATR spectroscopic investigation of organic carbon speciation in soils. Soil Science Society of America Journal, 69(1), 107-119.

Vivanco, J. C., Bojórquez, J. I., Murray, R. M., Nájera, O., Flores, F., \& Hernández, A. (2010). Características de los principales suelos de la cuenca del río Mololoa, Tepic, Nayarit, México. Cultivos Tropicales, 31(1), 00-00.

Woche, S. K., Goebel, M. O., Mikutta, R., Schurig, C., Kaestner, M., Guggenberger, G., \& Bachmann, J. (2017). Soil wettability can be explained by the chemical composition of particle interfaces-An XPS study. Scientific reports, 7, 42877 .
Xiao, X., Sun, Q., Kim, Y., Yang, S. H., Qi, W., Kim, D., \& Park, Y. (2018). Exposure to permethrin promotes high fat diet-induced weight gain and insulin resistance in male C57BL/6J mice. Food and chemical toxicology, 111, 405-416.

Yang, G. S., Vázquez, P. P., Frenich, A. G., Vidal, J. M., \& Aboul-Enein, H. Y. (2004). Separation and simultaneous determination of enantiomers of tau-fluvalinate and permethrin in drinking water. Chromatographia, 60(9-10), 523-526. 